\title{
Bicycle injuries among the paediatric population at an emergency department in Singapore
}

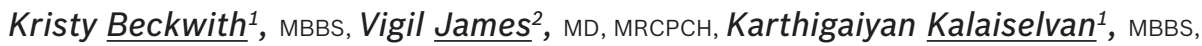
Sashikumar Ganapathy ${ }^{2}$, MB BCh BAO, MRCPCH

INTRODUCTION Bicycles injuries are the leading cause of emergency department visits among all recreational activities and have been established as a significant worldwide public health burden. The purpose of this retrospective study was to describe the epidemiology and patterns of paediatric bicycle-related injuries in Singapore.

METHODS This was a single-centre retrospective study based on data collected from a trauma registry between 2011 and 2016.

RESULTS A total of 760 patients presented to the emergency department during this period, of whom $68.2 \%$ were male and $39.7 \%$ were aged $10-16$ years. $50.1 \%$ of the patients were Chinese and $25.9 \%$ were Malay. The cyclist was the person most commonly injured (65.7\%) during the bicycle accident. From 2011 to 2015, the number of bicycle injuries showed a persistent upward trend. Superficial injuries such as abrasions and bruises were the most common injuries sustained $(46.1 \%)$, followed by fractures. The majority of the patients were discharged from the emergency department. There were no bicycle accident deaths recorded during the study period.

CONCLUSION Our study demonstrated an increasing number of bicycle injuries among paediatric cyclists in the local setting. Parents, teachers, coaches and other caregivers should be educated regarding the need to use protective equipment and to seek medical care promptly in the case of trauma resulting from bicycle injuries.

Keywords: bicycle injury, bicycling, children, public health, Singapore

\section{INTRODUCTION}

Among injuries from all recreational activities, bicycle injuries are the leading cause of emergency department (ED) visits. ${ }^{(1)}$ Injuries to cyclists have been established as a significant worldwide public health burden. ${ }^{(2)}$ This is of concern in Singapore, as cycling is a popular form of recreation, exercise and transport.

These injuries are mainly due to falls from bicycles and collisions with moving or fixed objects. Fatal and serious injuries are mainly the result of collisions with motor vehicles. ${ }^{(3,4)}$ Among the paediatric population, children aged 10-15 years have the highest fatality rates. ${ }^{(3)}$ While fracture is the major cause of morbidity in children, traumatic brain injury is the leading cause of mortality and long-term disability. ${ }^{(2,4)}$ In addition to this, handlebar accidents resulting in abdominal and pelvic organ injuries also result in a considerable amount of morbidity. ${ }^{(5)}$ Children who ride in bicycle-mounted seats are also at risk for injury, ${ }^{(6)}$ such as when their feet, legs or clothes get caught in the spokes of the wheel. ${ }^{(7)}$

Wearing bicycle helmets is the single most effective measure for prevention of significant bicycle injuries, particularly child bicycle helmets, which have the highest cost-benefit ratio among all injury prevention interventions. ${ }^{\left({ }^{8}\right)}$ As the number of bicycle injuries remains high despite many education programmes aimed at preventing bicycle-related injuries, this retrospective study aimed to describe the patterns of paediatric bicycle-related injuries.

\section{METHODS}

This was a single-centre retrospective study based on data collected at the children's ED at KK Women's and Children's Hospital, Singapore, between 2011 and 2016. This facility was chosen primarily because it is the biggest children's ED in Singapore and manages the majority of children (aged $\leq 16$ years) involved in accidents in Singapore. Our data was taken from the trauma registry and included all trauma-related injury, treatment, hospitalisations and deaths at the ED. This study was approved by the SingHealth Centralised Institutional Review Board hospital ethics committee.

All data was recorded by trained medical personnel at the $\mathrm{ED}$, including patient demographics, date and time of injury, place of occurrence, injury circumstances, mechanism of injury, interventions done at the ED and, subsequently, the patient's disposition. The mechanism of injury was categorised as follows: (a) fall; (b) direct collision with a bicycle; (c) road traffic accident; or (d) injury caused by a bicycle component. 'Yes' and ' $\mathrm{No}^{\prime}$ variables were used to code interventions performed at the ED, which included radiological imaging, the need for toilet and suturing, manipulation and reduction, cast immobilisations, and the activation of Code Blue and trauma code. Data was entered in Microsoft Excel 2016 (Microsoft Corp, Redmond, WA, USA). SPSS Statistics version 22.0 (IBM Corp, Armonk, NY, USA) was used to generate descriptive data for reporting. Data was shown as number of cases and percentage.

${ }^{1}$ Formerly NUS Yong Loo Lin School of Medicine, National University of Singapore, ${ }^{2}$ Children's Emergency, KK Women's and Children's Hospital, Singapore Correspondence: Ms Kristy Beckwith, Medical Officer, Tan Tock Seng Hospital, 11 Jalan Tan Tock Seng, Singapore 308433. Kristy.beckwith@mohh.com.sg 
Table I. Demographics of the patients with bicycle injuries $(n=760)$.

\begin{tabular}{|ll|}
\hline Variable & No. $(\%)$ \\
\hline Gender & \\
\hline Male & $518(68.2)$ \\
\hline Age (yr) & $242(31.8)$ \\
\hline$<1$ & $2(0.3)$ \\
\hline $1-2$ & $60(7.9)$ \\
\hline $3-4$ & $137(18.0)$ \\
\hline $5-9$ & $259(34.1)$ \\
\hline $10-16$ & $302(39.7)$ \\
\hline Ethnicity & \\
\hline Chinese & $381(50.1)$ \\
\hline Malay & $197(25.9)$ \\
\hline Indian & $102(13.4)$ \\
\hline Others & $80(10.5)$ \\
\hline Country of origin & \\
\hline Singapore & $570(75.0)$ \\
\hline China & $24(3.2)$ \\
\hline Philippines & $14(1.8)$ \\
\hline India & $53(7.0)$ \\
\hline Indonesia & $8(1.1)$ \\
\hline Malaysia & $17(2.2)$ \\
\hline Others & $74(9.7)$ \\
\hline Bicycle injuries by yr & 239 \\
\hline 2011 & 16 \\
\hline 2012 & \\
\hline 2013 & 230 \\
\hline 2014 & \\
\hline 2015 & \\
\hline 2016 (January-April) & \\
\hline
\end{tabular}

\section{RESULTS}

A total of 760 patients presented to the ED during this period. The distribution of bicycle injuries showed a male predominance, with $68.2 \%$ of the injuries occurring among the male paediatric population (Table I). Patients were mostly aged 10-16 years (39.7\%), closely followed by the 5-9 years age group (34.1\%). Half of the injured children were Chinese.

The number of bicycle injuries showed a uniform distribution throughout the months of the year (Fig. 1). There was an increase in the number of bicycle injuries from year to year, which translated to an increment of $102.3 \%$ in 2013, 29.2\% in 2014 and $3.9 \%$ in 2015 (Table I). More injuries occurred in the evening and night when compared to the day $(71.8 \%$ vs. $25.3 \%)$. Two times the number of injuries happened on weekdays (Monday to Friday) compared to weekends (Saturday and Sunday).

Superficial injuries (i.e. abrasions and bruises) were the most common type of injury sustained during bicycle accidents $(46.1 \%)$. This was followed by fractures $(19.2 \%)$. The percentage of intra-abdominal injuries due to handlebars, a serious injury specific to bicycle accidents, was $1.4 \%$ (Fig. 2). Other injuries that were seen included lacerations; haematoma

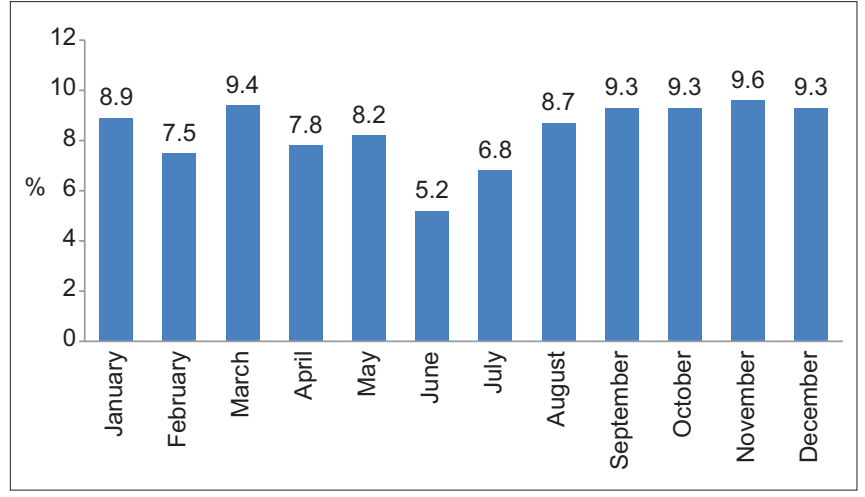

Fig. 1 Graph shows distribution of the bicycle injuries by month

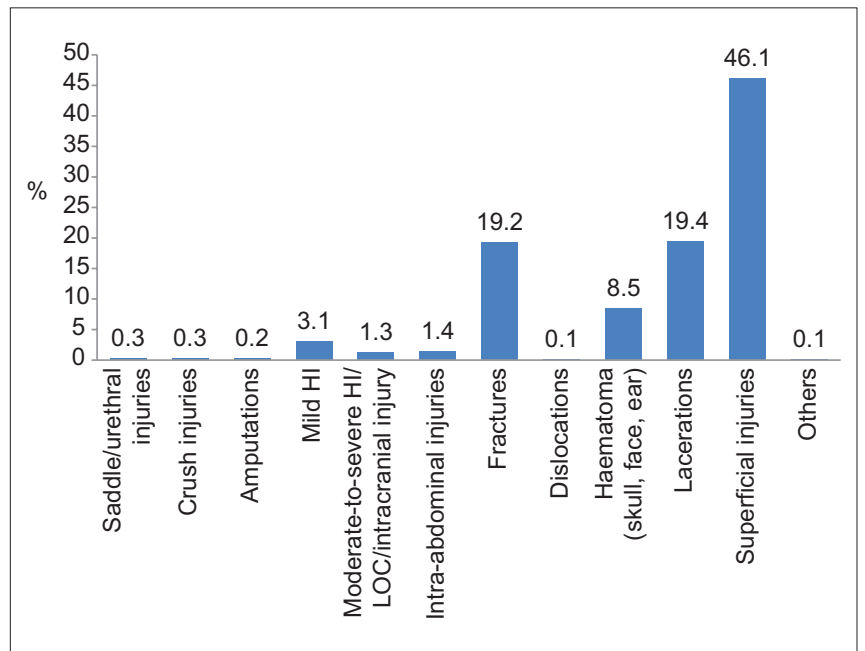

Fig. 2 Graph shows the types of bicycle injuries seen among the patients. $\mathrm{HI}$ : head injuries; LOC: loss of consciousness

of the skull, face and ear; dislocations; amputations; crush injuries; and saddle or urethral injuries. The upper (23.9\%) and lower limbs (42.3\%) were the most common anatomical areas injured during bicycle accidents, while the next commonest were those of the face (12.9\%) and head (9.6\%) (Fig. 3). Public places such as passages, footways, squares and forest reserves $(56.5 \%)$, roads and streets $(21.6 \%)$, residential institutions $(9.4 \%)$ and playgrounds $(7.5 \%)$ were the predominant sites where bicycle injuries occurred (Fig. 4). The person most commonly injured during the accident was the cyclist $(65.7 \%)$ and the next commonest person was the pillion rider (31.5\%). The impact with parts of the bicycle such as the spoke and wheel $(24.6 \%)$ resulted in the majority of the injuries. The handlebar $(3.6 \%)$ was the next most common part that was responsible for the injury.

The majority of the children (60.0\%) were discharged from the ED, to be followed up in surgical specialty clinics (Fig. 5). The next largest group (16.8\%) needed inpatient admission to a surgical specialty ward for ongoing operative management, consisting of open reduction and internal fixation of fractures, evacuation of intracranial haematomas, complex laceration repair, exploratory laparotomy, tissue debridement and eye injury repair. 6.6\% of the patients needed admission to the high dependency unit and intensive care unit, and $15.7 \%$ were discharged without 


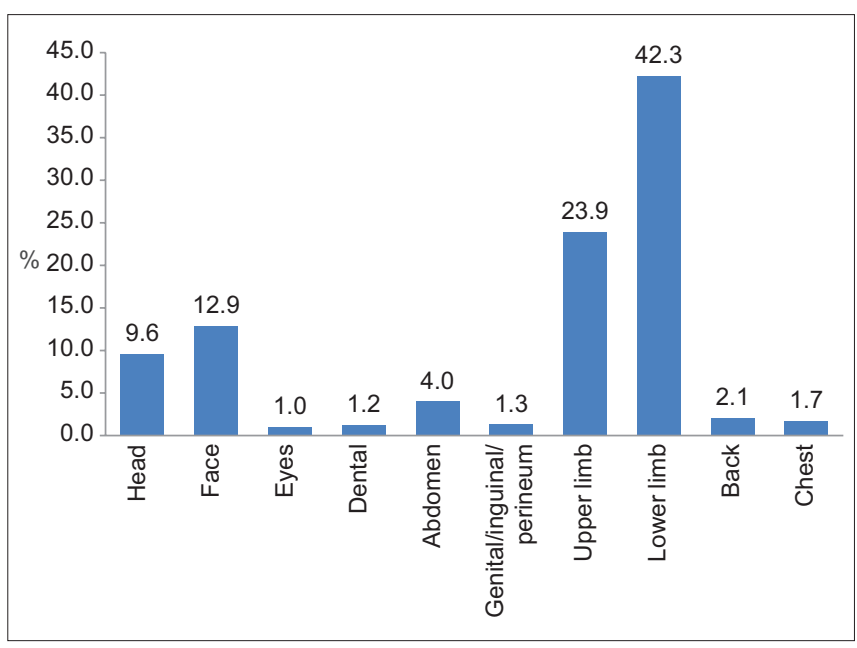

Fig. 3 Graph shows the anatomical distribution of the bicycle injuries.

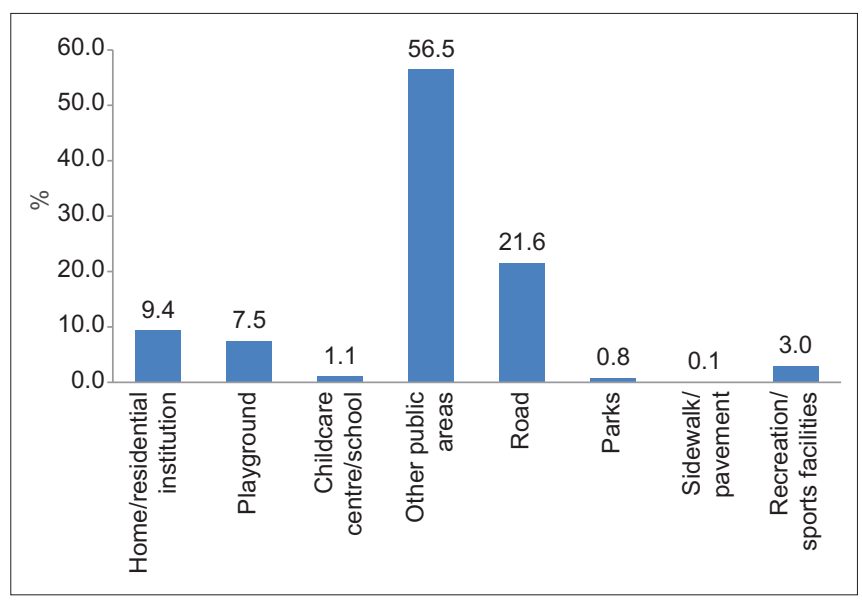

Fig. 4 Graph shows the locations where the bicycle injuries occurred.

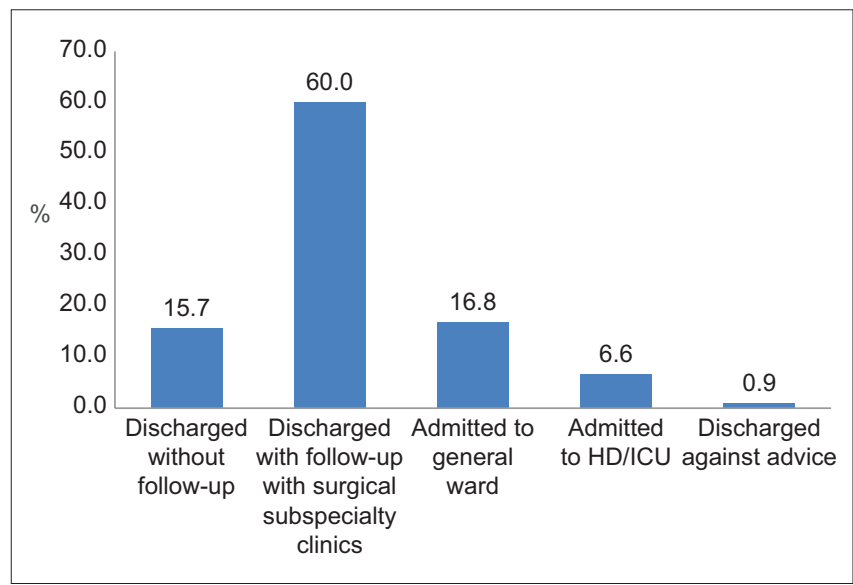

Fig. 5 Graph shows the disposition of the patients with bicycle injuries who presented to the emergency department. HD/ICU: high dependency/ intensive care unit

any follow-up. Children with head, abdominal and extremity injuries had more significant morbidity requiring hospitalisation and surgical interventions. No bicycle accident-related deaths were recorded between 2011 and 2016 among the patients who attended the ED (Fig. 5). Out of 760 patients, 5 (0.7\%) patients needed urgent computed tomography performed at the ED. None of the patients needed advance airway placement, but $5.5 \%$ needed blood product infusion at the ED.

\section{DISCUSSION}

The number of bicycle injuries showed an increasing trend during the study period. This concurred with the rise in trauma cases due to road traffic accidents in Singapore during this period. ${ }^{(9)}$ The predominance of male patients in our study was similar to that reported in previous studies. ${ }^{(4,10)}$ The presence of higher rates of trauma in male patients is attributed to their increased risk-taking behaviour and disproportionate engagement in outdoor activity. The peak age group for bicycle injuries reported in our study, 10-16 years, was also similar to the findings of other studies. ${ }^{(2)}$ This could be a result of various characteristics of this age group, such as increased speed in riding bicycles, exposure to roads where motor vehicles were concurrently present and lack of use of safety measures. ${ }^{(11)}$ The finding that three-quarters of the children were Singaporean is likely to be because the study was performed in Singapore, where there are more Singaporeans than non-Singaporeans, thus increasing their rate in the study cohort.

In our study, bicycle injuries predominantly occurred during the dusk and night periods. This result implies that the paediatric population should be strongly discouraged from cycling at dusk or after dark. ${ }^{(12,13)}$ When absolutely necessary, the rider should use protective measures such as reflective clothing and bicycle headlights. ${ }^{(12,13)}$ Additionally, the predominance of superficial injuries (i.e. abrasions and bruises) and lacerations among the study population has been reported in previous studies. ${ }^{(14)}$ The next most common injury type, namely fractures of the upper and lower extremities, was also similar to previous reports. ${ }^{(10,14,15)}$ The percentage of handlebar injures in our study $(1.8 \%)$ is similar to the $1 \%-2 \%$ in other studies. ${ }^{(5,16)}$ Although the number of handlebar injuries was low, the attending emergency medicine physician should not overlook this type of injury, as serious complications (e.g. splenic, liver or genitourinary injuries) can be an immediate outcome of such a traumatic injury. ${ }^{(16)}$ In terms of head injuries, the percentage in our study $(12.1 \%)$ was lower than those seen in the literature $(33.7 \%){ }^{.}{ }^{(2,6)}$ However, we should still emphasise the role of caregivers and physicians in counselling the paediatric population on the use of bicycle safety measures, especially the helmet, to further decrease the risk of injury.

The fact that the majority of the bicycle injuries occurred in public places (e.g. passages, footways, squares and forest reserves) emphasises the importance of developing dedicated riding areas such as bicycle lanes, especially for the paediatric age group who may not have adequate coordination skills to ride among fast-moving motor vehicles. ${ }^{(17)}$ Similar to previous studies, we found that the cyclist sustained the majority of the injuries in bicycle accidents, ${ }^{(9)}$ with the pillion rider being the next most commonly injured, although this was most likely because most of the cyclists involved did not have pillion riders. Strategies to prevent bicycle injuries should therefore focus on the safety of both the cyclist and the pillion rider. ${ }^{(2,6)}$

To lower the rates of bicycle-related injury, it is important to consider the use of passive preventive strategies and their success in injury prevention. These strategies, which have been suggested for other child-related products such as baby walkers, ${ }^{(18)}$ do not require constant vigilance or frequent monitoring by the user 
of the product. For example, wheels with spoke covers could prevent extremities from getting caught in the spokes $^{(19)}$ and energy-absorbing handlebars are less likely to cause abdominal injuries. ${ }^{(20)}$ These available modifications would likely reduce the number of bicycle-related injuries if their use were more widespread. Bicycle-related injuries require consultation across various specialties such as orthopaedics, general surgery, radiology, laboratory services and blood banks, placing an additional burden on healthcare resources.

For healthcare providers, mainly emergency physicians and paediatricians, knowledge of injury patterns provides a framework for preventative education. Based on our findings, the most appropriate preventative measures appear to be efforts to encourage supervised cycling and the use of appropriate protective equipment. A multi-tiered public health approach that targets education, enforcement and legislation, and engineering offers the most effective strategy for injury prevention. ${ }^{(21)}$ Emphasis should be placed on learning the rules of the road such as riding with traffic, stopping and looking both ways before entering the street, stopping at all intersections, and using hand signals before turning. Young cyclists should be also advised to use bicycle paths whenever possible, avoid riding at dusk or after dark, avoid wearing earphones while cycling, and place objects in their backpack or basket.

This study was not without limitations. Our findings may not be representative of the true number of bicycle injuries in Singapore, as patients treated in other healthcare settings and those who did not receive medical attention were not included in the surveillance database. The trauma registry is a surveillance tool that, by definition, does not furnish a complete set of data, collecting data only from hospital-treated patients. A better methodology would be to retrieve records based on ICD-10 (International Classification of Diseases-10) codes or discharge diagnoses. It would also have been useful to study the use of safety measures such as helmets and the number/percentages of children with Injury Severity Score $\geq 16$, but this data was not consistently available. We also could not know how many discharges did not require ED-level care (e.g. no radiological examination or long observation) and whether the visit could have been avoided with appropriate first aid care or presentation to a general practitioner, as this data was similarly not available. Likewise, it would be useful to collect longer-term data on the patients with bicycle injuries in order to prognosticate long-term outcomes in these patients.

In conclusion, our study demonstrated an increasing number of bicycle injuries in children in the local setting. As both the cyclist and the pillion rider are at risk of injury, protective measures should be used by both parties. The vast majority of injuries sustained were minor, superficial limb injuries, followed by fractures of the long bones, with no deaths noted. Additionally, the majority of the patients were discharged from the ED with close follow-up at the orthopaedic clinic. A multitiered public health approach is the most effective strategy for injury prevention, including educating caregivers on the importance of their child's use of helmets, enforcement and legislation to mandate following traffic rules while cycling, the use of safety measures, and engineering efforts to manufacture wheels with spoke covers and energy-absorbing handlebars. ${ }^{(21)}$ Discouraging children from cycling after dark and encouraging the use of reflective clothing, bicycle headlights and dedicated bicycle lanes for cycling are other useful measures that can be implemented.

\section{REFERENCES}

1. Injury-control recommendations: bicycle helmets. National Center for Injury Prevention and Control, Centers for Disease Control and Prevention. MMWR Recomm Rep 1995; 44(RR-1):1-17.

2. Shah S, Sinclair SA, Smith GA, Xiang H. Pediatric hospitalizations for bicyclerelated injuries. Inj Prev 2007; 13:316-21.

3. Acton $\mathrm{CH}$, Thomas $\mathrm{S}$, Nixon JW, et al. Children and bicycles: what is really happening? Studies of fatal and non-fatal bicycle injury. Inj Prev 1995; 1:86-91.

4. Puranik S, Long J, Coffman S. Profile of pediatric bicycle injuries. South Med J 1998; 91:1033-7.

5. Winston FK, Weiss HB, Nance ML, et al. Estimates of the incidence and costs associated with handlebar-related injuries in children. Arch Pediatr Adolesc Med 2002; 156:922-8.

6. Powell EC, Tanz RR. Cycling injuries treated in emergency departments: need for bicycle helmets among preschoolers. Arch Pediatr Adolesc Med 2000; 154:1096-100.

7. Powell EC, Tanz RR. Tykes and bikes: injuries associated with bicycle-towed child trailers and bicycle-mounted child seats. Arch Pediatr Adolesc Med 2000; 154:351-3

8. Ginsberg GM, Silverberg DS. A cost-benefit analysis of legislation for bicycle safety helmets in Israel. Am J Public Health 1994; 84:653-6.

9. Chong SL, Tyebally A, Chew SY, et al. Road traffic injuries among children and adolescents in Singapore - who is at greatest risk? Accid Anal Prev 2017; 100:59-64

10. Brown RL, Koepplinger ME, MehIman CT, Gittelman M, Garcia VF. All-terrain vehicle and bicycle crashes in children: epidemiology and comparison of injury severity. J Pediatr Surg 2002; 37:375-80.

11. Road traffic injuries. In: Peden M, Oyegbite K, Ozanne-Smith J, et al, eds. World Report on Child Injury Prevention. Geneva: World Health Organization, 2008.

12. Schor EL. Caring for Your School Age Child: Ages 5-12 (Child Care S). Bantam; Rev Sub edition, 1999.

13. Thompson MJ, Rivara FP. Bicycle-related injuries. Am Fam Physician 2001; 63:2007-14.

14. Ortega HW, Shields BJ, Smith GA. Bicycle-related injuries to children and parental attitudes regarding bicycle safety. Clin Pediatr (Phila) 2004; 43:251-9.

15. Frank E, Frankel P, Mullins RJ, Taylor N. Injuries resulting from bicycle collisions. Acad Emerg Med 1995; 2:200-3.

16. Clarnette TD, Beasley SW. Handlebar injuries in children: patterns and prevention. Aust N Z J Surg 1997; 67:338-9.

17. Mehan TJ, Gardner R, Smith GA, McKenzie LB. Bicycle-related injuries among children and adolescents in the United States. Clin Pediatr (Phila) 2009; 48:166-73.

18. Smith GA, Bowman MJ, Luria JW, Shields BJ. Babywalker-related injuries continue despite warning labels and public education. Pediatrics 1997; 100:E1.

19. D'Souza LG, Hynes DE, McManus F, et al. The bicycle spoke injury: an avoidable accident? Foot Ankle Int 1996; 17:170-3.

20. Arbogast KB, Cohen J, Otoya L, Winston FK. Protecting the child's abdomen: a retractable bicycle handlebar. Accid Anal Prev 2001; 33:753-7.

21. Yanchar NL, Warda LJ, Fuselli P. Child and youth injury prevention: a public health approach. Paediatr Child Health 2012; 17:511-2. 